\title{
Mujeres y violencia transgeneracional: mitos y creencias que naturalizan el maltrato en los sistemas familiares ${ }^{1}$
}

\author{
Mª Elizabeth González Borbarán*
}

\begin{abstract}
RESUMEN
El presente artículo da cuenta de los resultados de un estudio de casos que permitió conocer las creencias y mitos que mujeres con vivencias abusivas en su historia, le atribuyen a las dinámicas familiares con violencia, maltrato y abuso sexual, y las explicaciones que éstas se dan con respecto a la naturalización de estas pautas transmitidas transgeneracionalmente. Con el propósito de develar las explicaciones que las mujeres entrevistadas le otorgan a la ocurrencia y repitencias de situaciones abusivas, se utilizó un esquema básico de análisis estructural semántico, logrando de este modo una representación simbólica de sus relatos, los cuales están sometidos a ciertas reglas y códigos que son inherentes a su contexto y sobre el cual configuran sus explicaciones de la vida cotidiana.
\end{abstract}

Palabras Clave: Mujeres - violencia - transmisión transgeneracional - mitos - Creencias - naturalización - sistemas familiares.

\section{Mulheres e violência transgeneracional: Mitos e crenças que naturalizan o maltrato nos sistemas familiares}

\section{RESUMO}

O presente artigo dá conta dos resultados de um estudo de casos que permitiu conhecer as crenças e mitos que mulheres com vivências abusivas em sua história, lhe atribuem às dinâmicas familiares com violência, maltrato e abuso sexual, e as explicações que estas se dão com respeito à naturalización destos padrões transmitidos transgeneracionalmente. Com o propósito de develar as explicações que as mulheres entrevistadas lhe outorgam à ocurrencia e repitencias de situações abusivas, se utilizou um esquema básico de análise estrutural semántico, conseguindo deste modo uma representação simbólica de seus relatos, os quais estão submetidos a certas regras e códigos que são inherentes a seu contexto e sobre o qual configuran suas explicações da vida quotidiana.

Palavras chave: Mulheres - violência - transmissão transgeneracional mitos - crenças - naturalización - sistemas familiares.

1 Este artículo ha sido elaborado sobre la base de la tesis realizada durante el año 2010, para optar al grado de Magíster en Intervención Social, mención Familias, impartido en la Universidad Católica Silva Henríquez, Chile. Profesora guía: Sandra Iturrieta Olivares.

* Chilena. Trabajadora Social. Magíster en Intervención Social. Asistente Social de Consejo de Defensa del Niño (CODENI). Correo electrónico: eliborbaran@ gmail.com 


\title{
Women and violence transgeneracional: Myths and beliefs that naturalizan the maltreatment in the familiar systems
}

\author{
ABSTRACT
}

This article reports the results of a case study yielded information on the beliefs and myths that women with abusive experiences in their history, attributed to family dynamics with violence, abuse and sexual abuse, and the explanations they are given regarding the naturalization of these guidelines transgenerationally transmitted. In order to reveal the women interviewed explanations give to the occurrence and repetition of abusive situations, we used a basic semantic structural analysis, thereby achieving a symbolic representation of his stories, which are subject to certain rules and codes that are inherent in its context and which shape their accounts of everyday life.

Key words: Women - violence - transmission transgeneracional - myths Beliefs - naturalización - familiar systems. 


\section{Antecedentes}

Hacer referencia a la violencia intrafamiliar, necesariamente implica pensar en una situación de sufrimiento, más aún si en las últimas décadas en Chile se ha intentado distintas estrategias para erradicarla, sin embargo, los índices demuestran que aún se está lejos de terminar con ella. La sociedad chilena se ha transformado y ha dado indicios al mundo de que es un país que crece y se moderniza, no obstante, este progreso no se condice con la cultura que sigue reproduciendo patrones en la sociedad que permiten mantener y transmitir estilos relacionales basados en conductas abusivas y/o maltratadoras.

El fenómeno de la violencia se sustenta en el modelo cultural patriarcal, enmarcado en un contexto dominio/sumisión que interfiere y ordena las relaciones sociales entre hombres y mujeres a partir de una construcción relacional determinada por cuotas de poder asociadas a las diferencias de género, que implican una forma desigual de relacionarse y delimitan los espacios jerárquicos que sirven de barreras y límites excluyentes o de inclusión de personas o grupos de menor poder o subordinados, siendo las mujeres, los niños y niñas, quienes se ubican en estos grupos en el patriarcado, el que se caracteriza por definir roles, funciones, posiciones y jerarquías dentro de la sociedad según el género.

En este contexto la violencia surge como una de las formas de representación del patriarcado, instalado y sustentado en patrones culturales que se transmiten como un modo socialmente aceptado de relacionarse, provocando que se establezcan rasgos en la socialización masculina y femenina que opera por una parte, como un mecanismo de resolución de conflictos y por otro lado, perpetúa la subordinación de los grupos con menor poder, generando con esto un desequilibrio en la relación e imponiendo el poder con el uso de la fuerza.

Las pautas de comportamiento que sustentan este patrón, se sitúan de tal manera que se van familiarizando como una relación normalizada por el propio sistema “...se instauran formas aprendidas de relacionarse, que tienen que ver con las pautas de crianza, disfuncionalidad o estructura de la familias" (Larraín, 2002:36). Es así como las pautas de crianza adquieren mayor fuerza en la red vincular más próxima a las personas que es la familia, con patrones que se trasmiten generacionalmente 
y que se apropian de los espacios de interacción cruzando transversalmente distintos ámbitos y niveles internos y externos de las familias.

La repetición de patrones transmitida de generación en generación, se relaciona con la representación cultural que tienen los integrantes de una familia. Según Barudy (2001), la noción de cultura vista desde las familias, podría definirse como el conjunto de "...conductas que permanecen constantes a través de las generaciones (...) de discursos y/o relatos que se trasmiten y se mantienen de generación en generación y que dan sentido y explican los acontecimientos y fenómenos de la vida familiar" (Barudy, 2001:44). A partir de estas representaciones y discursos sociales, que sirven de referencia a los integrantes de un grupo familiar, se van moldeando ciertas pautas de comportamiento, roles, estereotipos y estilos de relacionarse. Desde la perspectiva transgeneracional las situaciones de violencia se asumen como un aprendizaje que se adquiere en la familia de origen; si este núcleo familiar fue un espacio con situaciones abusivas o maltratadoras las creencias y patrones de naturalización con respecto a la violencia se transmitieron.

Al transmitir esta conducta a las nuevas generaciones, la violencia se naturaliza como un estilo de relación legítimo para el sistema familiar, aprendido como un modelo de convivencia que presenta un repertorio conductual que busca según las creencias del sistema, mantener el equilibrio entre lo que cada miembro aporta o recibe, internalizando y legitimando normas y pautas (Palma, 2007). La interacción entre sus integrantes se estructura a partir de un estilo jerárquico rígido, reproductor de desigualdad, con creencias estereotipadas y poco flexibles en relación al género, que se adquiere de las representaciones dadas por un entorno mayor que influye en la prevalencia de ciertas creencias con respecto a lo femenino y masculino: “. . la violencia familiar desde la perspectiva de las relaciones de abuso y, en el marco del pensamiento sistémico, desarrolla un esquema que permite por un lado, entender cómo se mantienen las relaciones abusivas y, por otro lado, sirve de guía para producir una perturbación en ella" (Ravazzola, 1997:9), entendido lo anterior a partir de la concepción de un sistema que interactúa con otros, que tiene influencias de un sistema mayor y que también está determinado por ese contexto inmediato y dominante.

La realidad de las familias se caracteriza hoy por su multiplicidad y diversidad, lo que ha implicado mirarlas desde distintas 
perspectivas y enfoques, a fin de considerar los cambios que han tenido en las últimas décadas en el ámbito de su estructura, como en tanto unidad social afectada por las transformaciones e influencias de la globalización y de la postmodernidad, que han generado cambios en su dimensión cultural, imponiendo una nueva concepción de valores, creencias y representaciones.

El conflicto se presenta como parte de un proceso interactivo interno y actúa como sistema de control en este tipo de familias con dinámicas abusivas, manifestándose en violencia intrafamiliar. (Iturrieta, 2001). Las diferencias exteriorizadas en conductas maltratadoras podrían ser una reproducción y reflejo de los conflictos sociales que se traspasan al ámbito familiar, naturalizando este tipo de aprendizaje. Algunos autores como Gracia y Musitu (2000) han desmitificado la idea de las familias como un todo armonioso, dado que conviven personas con discrepancias que tienen relación con sus propias creencias y supuestos acerca de la familia. Klein y While (1996) señalan que "Las familias tienen una naturaleza paradójica, puesto que existe conflicto intenso (...) donde coexisten antagonismos, el odio, la violencia y el apoyo (... quizás donde se refleja con mayor nitidez esta naturaleza paradójica de las familias, sea el de la violencia intrafamiliar, donde coexisten el amor, el miedo, la vergüenza y en ocasiones el odio" (Klein 1996, citado en Iturrieta, 2001:17)

Toda familia tiene una estructura que le permite sobrevivir y alcanzar sus metas y objetivos, sin embargo, es necesario hacer una diferenciación entre una familia sana y aquellas con dinámicas maltratadoras, las cuales cambian los recursos esenciales que están al servicio del bienestar integral del grupo familiar, generando conflictos, conductas que pueden estar asociadas a las conexiones que hacen los adultos con sus propias historias de vida, sus experiencias de infancia y de la forma en que fueron abordados los problemas en sus familias de origen, lo que podría incidir en la eventualidad de bloquear o disminuir la posibilidad de autorregulación frente a los conflictos, tomando en cuenta que los recursos adecuados están momentáneamente agotados.

Lo anterior, se relaciona con los sistemas de creencias, aprendizajes, normas y reglas que fueron adquiridas en la infancia y que se acuñan en la actual cotidianidad familiar, caracterizada por "...el encierro intrafamiliar que facilita la acumulación de tensiones internas y/o la ausencia de un cuestionamiento crítico de los sistemas de creencias abusivos y violentos" (Barudy, 2001:43). 
En las familias donde priman las tensiones y desequilibrios, la ejecución y obligación de llevar a cabo ciertos mandatos va a requerir de una exigencia superior, sumándose a esto las obligaciones y requerimientos provenientes de generaciones anteriores. En un sistema familiar con relaciones sanas estos requerimientos están claros y generan un ambiente de confianza básica entre los integrantes de la familia. Sin embargo, cuando hay ausencia o poca claridad en las reglas y normas, se produce el caos y por ende el conflicto, que por lo general resulta en imposición de la autoridad a través de métodos violentos y maltratadores.

Las familias con dinámicas violentas, aquellas que crean y establecen lealtades insanas, no logran sustentar, contener o apoyar adecuadamente en un momento de crisis familiar, moviéndose en una constante oscilación entre la disminución y el aumento de la vitalidad de cada sistema familiar, asociado a las historias multigeneracionales de las familias que finalmente van desgastando el mundo interno de estas y de sus integrantes, provocando culpas y frustraciones que podrían desembocar en una escalada aún más maltratadora y abusiva. Esto porque los mecanismos defensivos se van transformando en las formas de enfrentar los conflictos, sin embargo, estas pautas logran que el sistema vuelva al equilibrio de manera indirecta; es lo que Boszormenyi-Nagy llama las lealtades invisibles. (BoszormenkyiNagy, 1982:54)

Las relaciones interpersonales de tipo abusivo se asocian principalmente a desigualdades en las relaciones, a partir de la concepción que tengan los integrantes de un sistema familiar, con respecto de las creencias y de los mitos acerca del mundo masculino y femenino. En las familias maltratadoras el modelo cultural determina a partir de un mandato socialmente aceptado, la discriminación, la desigualdad, el estereotipo de roles rígidos que se va reproduciendo en este sistema abusivo. Los integrantes del sistema familiar tienen este estilo de relación concebida por este ordenamiento socialmente dado que replica las dinámicas abusivas, las reproduce sin mediar cuestionamientos ni resistencias de parte de las víctimas. Las conductas se perpetúan degradando lo femenino en relación a lo masculino, estableciendo un sistema jerárquico y rígido con respecto de los roles, que se instalan para preservar esta jerarquía de superioridad al interior del vínculo. 
Estas dinámicas se dan como forma de organización que se ha establecido a partir de ciertas creencias que van modelando conductas, ideas y estilos de relación, influidas por lo que Barudy (2001) denomina ideología patriarcal y que se entiende como “... el aprendizaje de la obediencia y la sumisión a la autoridad del hombre" (Barudy, 2001:117). A partir de esta aseveración, se puede deducir que los postulados patriarcales afirman la perspectiva de superioridad de lo masculino por sobre lo femenino, de la desvalorización construida en la base de la creencia de percibir a las mujeres como fracasadas y a los hombres valorizados por su autoridad y autonomía.

La perspectiva transgeneracional permite entender estos procesos de socialización, debido a que en esta unidad social (la familia) se realiza el aprendizaje de la transmisión cultural, de patrones de parentesco, del estilo de vida familiar, entre otros de la familia de origen. Colocando el énfasis en describir estos procesos de transmisión de creencias, comportamentales o emocionales a través de las generaciones. Para Bowen (1981) estos procesos de transmisión, están ligados no sólo al momento actual de las familias, sino están relacionados con el pasado a través de la transmisión multigeneracional.

La transmisión representa el conjunto de normas, valores, mitos e identidad que se socializan en el ámbito de las relaciones familiares y se traspasan de una generación a otra. Al hacer referencia a sistemas familiares con dinámicas violentas y/o abusivas, se está sosteniendo que esta es una situación heredada donde los modelos, mitos y creencias, surgen y se aprenden en la familia de origen, siendo las manifestaciones de violencia o abuso, expresiones de historias de sufrimiento y dolor de los adultos que repiten este tipo de pautas de conductas aprendidas.

\section{Metodología}

La investigación se enmarcó en el paradigma interpretativo, dado que se esperaba conocer las creencias que las mujeres otorgaban a sus propias experiencias de violencia transgeneracional, comprendiendo desde sus relatos cuáles eran las creencias asociadas a la naturalización de las conductas maltratadoras que se repiten transgeneracionalmente en sus actuales familias.

Las mujeres informantes correspondieron a: abuelas, madres y nietas en cuyas narrativas existieran experiencias de violencia, 
maltrato y/o abuso sexual transgeneracional por a lo menos tres generaciones. Estos criterios de selección se determinaron a partir del supuesto de que en las familias se establecen tipos de vínculos que se traspasan transgeneracionalmente. Al investigar tres generaciones de mujeres de una misma familia, se podría dilucidar las creencias que sustentan la naturalización de las situaciones abusivas.

Con entrevistas en profundidad se recogieron los relatos de estas mujeres, siendo posteriormente realizado un análisis longitudinal que permitió identificar las categorías y un análisis transversal que estableció las subcategorías, las que fueron analizadas para conocer las explicaciones dadas por esas mujeres a su situación, a través de un análisis estructural semántico.

Las categorías que emergieron de las narrativas de las generaciones de las abuelas, madres y nietas, fueron el sufrimiento, la violencia, el maltrato y la negligencia, como situaciones abusivas que cruzaron transversalmente la vida de estas mujeres.

Cuadro $\mathrm{N}^{\circ} 1$. Categorías y sub-categorías de análisis

\begin{tabular}{|l|l|}
\hline $\begin{array}{l}\text { CATEGORÍA 1: SUFRIMIENTO } \\
\text { Se entenderá como un estado } \\
\text { intenso de sentir desconsuelo, } \\
\text { angustia, padecer daño físico, } \\
\text { psicológico o moral. }\end{array}$ & Subcategoría 1.1: Tristeza \\
\cline { 2 - 2 } $\begin{array}{l}\text { CATEGORÍA 2: VIOLENCIA Y } \\
\text { MALTRATO }\end{array}$ & Subcategoría 1.2: Rabia \\
\cline { 2 - 2 } $\begin{array}{l}\text { Es toda acción intencional de causar } \\
\text { o inflingir daño a otro, por el solo } \\
\text { hecho de estar en una relación de } \\
\text { poder con respecto de ese otro. }\end{array}$ & Subcategoría 2.1: Física \\
\cline { 2 - 2 } $\begin{array}{l}\text { CATEGORÍA 3: NEGLIGENCIA } \\
\text { Se entenderá como una forma pasiva } \\
\text { de maltrato que puede ser visible } \\
\text { o invisible, en relación a descuido, } \\
\text { omisión, abandono. }\end{array}$ & Subcategoría 2.3: Abuso Sexual \\
\hline
\end{tabular}

Como hemos dicho, con la finalidad de establecer las explicaciones que las entrevistadas atribuyen a la ocurrencia de situaciones abusivas que se transmiten transgeneracionalmente, se aplicó un esquema básico de análisis estructural semántico a cada categoría, lo que implicó representar simbólicamente los relatos sometiéndolos a ciertas reglas y códigos que son propios de su contexto y sobre el cual, configuran sus explicaciones de la vida cotidiana. 
El análisis estructural semántico permite reconocer un contexto dado donde hay un sujeto que aspira a alcanzar un fin o un objeto valor, para lo cual cuenta con oponentes y adversarios que dificultan el logro de este objetivo. Por otra parte, coexisten los ayudantes y oponentes que facilitarán u obstaculizarán el proceso, el que requiere de acciones como mecanismos de salida, que permitan alcanzar el objeto valor, condicionado por la identidad del sujeto, versus la alteridad que también pueden obstaculizar o facilitar que el remitente logre el objeto valor y lo comparta con un destinatario.

\section{Principales resultados}

Respecto del sufrimiento, éste se constituye en el objeto valor que es no sufrir, para el logro del objetivo cuenta con ayudantes y adversarios, los primeros son los hombres que se configuran en la vía de escape al sufrimiento, los que en el futuro pasaran a convertirse en adversarios. Por otra parte, los adversarios se conforman de aquellos adultos a cargo, especialmente en su primera infancia, que las vulneran, y los sentimientos de soledad y culpa que las instan a salir de su estado de sufrimiento.

Para alcanzar el objeto valor, las mujeres realizan acciones como mecanismos de salida, tales como abandonar la familia de origen y casarse, esto les permitiría alcanzar el objeto valor que es no sufrir; este proceso estará condicionado a la identidad de las mujeres, es decir, cómo ellas se ven, versus la alteridad, o sea cómo las ven los otros. Lo anterior puede obstaculizar o facilitar que las mujeres, como remitentes, logren el objeto valor, que es dejar de sufrir, y lo compartan con un destinatario, que es dar una mejor vida a las hijas e hijos.

Las mujeres, con respecto al sufrimiento, transmiten la creencia de que las vivencias abusivas generan una emocionalidad vivida con desesperanza, que no se puede cambiar y que provoca un sufrimiento permanente. También, que las situaciones abusivas vulneran su condición de mujer, generando sentimientos de injusticia, lo que provoca sufrimiento; bajo la creencia de que la rabia es la emoción válida para expresar el sufrimiento, la tristeza es valorada negativamente como debilidad femenina, porque la rabia moviliza mientras que la tristeza paraliza.

En relación a la violencia y maltrato, las mujeres aspiran a alcanzar el objeto valor que es dejar de vivir violencia y maltrato, 
constituyéndose en los ayudantes para el logro del objetivo, el apoyo de sus madres, la existencia de los hijos e hijas y lograr la independencia. Los adversarios que obstaculizarán el logro del objeto valor, son los hombres agresores, la cultura patriarcal que determina ciertos aprendizaje y creencias. Los mecanismos de salida que ellas visualizan son responder a la violencia con más violencia, denunciar los abusos y separarse del agresor.

En relación a la violencia y el maltrato, se transmite la creencia de que creando estrategias como la sumisión femenina, se debería garantizar no estar expuesta a situaciones abusivas y al existir, es necesaria la búsqueda de mecanismos de escape que les permitan dejar de vivir situaciones abusivas. Existe la creencia de que el maltrato es una forma de enseñanza necesaria para la regulación de la dinámica familiar cuando viene de uno de los integrantes de la familia. También, que la obediencia ante las exigencias de los hombres evita conflictos que terminan en violencia.

Respecto a la negligencia, está esa atribuida a las mujeres, por tanto el objeto valor es dejar de serlo. Para con lo que cuenta con ayudantes que son pedir ayuda externa y que se reconozca la negligencia como un acto abusivo. Los adversarios que intentarán impedir que alcancen el objeto valor son la violencia, la pobreza, las creencias y la pasividad. Las estrategias y mecanismos de salida para dejar de ser negligentes, es contar con las abuelas que acompañan y madres que reparan. En este proceso las mujeres se ven, con respecto a la negligencia, como víctimas, preocupadas, sacrificadas y que mantienen la unidad familiar, versus como las ven los otros; malas madres, castigadoras, irresponsables, abandonadoras, indiferentes, cómplices y poco afectivas.

Las explicaciones que se dan las mujeres en relación a la negligencia son las creencias de que la sumisión del género femenino favorece la unión familiar no como algo que se valora, sino como ordenamiento dado. Asumen también que hay actos que no pueden ser contados pues conforman parte de los secretos de las familias. Además es una creencia en que la condición de mujer víctima y vulnerable está por sobre la de madre protectora, ya que sólo por la condición de ser madre, debe haber incondicionalidad en el vínculo.

Otras creencias asociadas son que desarrollando estrategias como la sumisión femenina, se debería garantizar no estar expuesta a situaciones abusivas, esto porque la obediencia ante las exigencias de los hombres evitaría conflictos que terminen en 
violencia. También existe la creencia de que el maltrato cuando viene de uno de los integrantes de la familia, es una forma de enseñanza necesaria para la regulación de la dinámica familiar, pero que es necesaria la búsqueda de mecanismos de escape que les permitan dejar de vivir situaciones abusivas. La negligencia frente a situaciones abusivas se atribuye a conductas relacionadas con el rol de la crianza, pero se relativiza cuando las mujeres se ven expuestas a situaciones abusivas.

\section{Discusión de resultados}

Al momento de reflexionar sobre los resultados de este estudio, es preciso señalar que los logros y alcances de la investigación realizada no habrían sido posibles sin la disponibilidad de las mujeres entrevistadas, que con sus historias de vida permitieron entender y profundizar en el rol que juegan los mitos y creencias y cómo estos se transmiten transgeneracionalmente marcando la vida de las descendencias.

Es necesario, para conocer estas creencias y mitos en relación al maltrato, considerar que este tema genera hoy diversas opiniones con respecto a su campo de intervención, dado que observar el fenómeno, reconocer y analizar sus causas y efectos no necesariamente ha implicado contar con las herramientas más adecuadas para abordarla de manera eficaz.

Es posible establecer a partir del análisis de los relatos de las tres generaciones de mujeres, que existen repitencias de pautas transgeneracionales directamente ligadas con situaciones abusivas en el contexto intrafamiliar. Las vivencias de violencia generan una emocionalidad vivida con desesperanza, por una condición que no se logra modificar siendo el sufrimiento un estado permanente en todas las mujeres entrevistadas y que cruza transversalmente todas sus etapas de vida: infancia, adolescencia y adultez, y se va transmitiendo a las siguientes generaciones de la misma manera, como desesperanza aprendida por todas las mujeres de una misma familia.

Las mujeres hacen intentos, a través de su historia, de detener su condición, a través de estrategias tales como la sumisión, dado que se tiene la creencia de que este sometimiento debería garantizar no estar expuesta al abuso y al maltrato. Ello se refuerza porque la realidad indica que el no acatamiento de las normas al interior de una familia con dinámica maltratadora, es considerado 
como una trasgresión a lo establecido e impuesto por quien tiene el poder dentro del sistema, lo que significará estar expuesta a sanciones de parte de el o los agresores. Se transmite por tanto, que el maltrato es una forma de enseñanza necesaria para la regulación de la dinámica familiar cuando viene de uno de los integrantes del sistema; no se vivencia del mismo modo cuando quien agrede es una persona ajena al núcleo familiar. Está en la base la creencia de que el derecho a sancionar es exclusivo de los adultos que conforman el grupo familiar, siendo esta creencia también traspasada transgeneracionalmente.

Se trasmite a las siguientes generaciones que es necesaria la búsqueda de mecanismos de escape, que les permita a las mujeres dejar de estar expuestas a este tipo de actos. En todos los relatos de las generaciones de las abuelas y las madres, la alternativa de escape a la violencia recibida en su entorno familiar, se va transmitiendo a las hijas y a su vez se les enseña a las nietas, lo que se contrapone con la transmisión de la desesperanza aprendida. Todo este proceso se vive en una permanente tristeza que se traslada de la infancia a la adolescencia y a la etapa adulta, especialmente si reconocen que la estrategia no logró los resultados esperados, viviéndola como un retroceso, pues se hizo una apuesta en un hombre que en un primer momento se vio como alguien que rescata, sin embargo, con el paso del tiempo, se convierte en un nuevo agresor. Se trasmite, por tanto, que hay situaciones que no pueden modificarse.

En las narrativas de las mujeres hay claridad en reconocer que la violencia se aprende, como forma de educar, de relacionarse, de resolver conflictos, y como defensa. Lo perciben y transmiten como un sentimiento de injusticia por el trato recibido, que vulnera su condición de mujer; esto provoca sufrimiento, el que se manifiesta en alguna etapa a través de la rabia, transmitiendo a las hijas y nietas que la pena es manifestación de debilidad; la rabia por tanto, moviliza, especialmente al constatar que las estrategias buscadas como mecanismo de escape fracasaron, estas emociones y deducciones son transmitidas a las generaciones siguientes a través de la palabra o con acciones. Se transmite fuertemente la creencia de que la rabia es la emoción válida para expresar un sufrimiento; sentir pena o tristeza se valora negativamente como debilidad femenina, todas las mujeres entrevistadas mezclan en sus narrativas la rabia con la pena, señalando que la primera moviliza, mientras que la segunda paraliza. 
Las situaciones vividas por las mujeres no son percibidas como formas naturalizadas de relacionarse, porque están en una constante búsqueda de salidas de esta condición, no logrando nunca adaptarse a este estilo relacional, siendo evidenciado por las generaciones siguientes. La aparente pasividad que se observa obedece a la sensación de temor constante, la no respuesta ante los abusos es una manera de protegerse; esta respuesta tiene mucho que ver con la etapa de vida en que se encuentran las mujeres. En el caso de las nietas, su condición de desigualdad frente a sus agresores las expone con mayor facilidad a malos tratos, la desobediencia a la autoridad puede significar para una niña ser objeto de agresiones. En las mujeres adultas, sin embargo, las maneras de enfrentar las situaciones abusivas son responder con violencia; es lo que refieren las abuelas y las madres, lo que las ubica en un sitial de mayor igualdad con su agresor, siendo esta conducta transmitida a las hijas y nietas. El uso de la fuerza tiene directa relación con la posición de poder dentro de un sistema familiar; este sometimiento se extrapola por sobre el vínculo aun cuando éste se haya terminado; la vulnerabilidad de las mujeres también se trasmite, la imagen femenina que se traspasa a las hijas y nietas es de dependencia y subordinación.

Se transmite además la obediencia ante las exigencias de los hombres, se traspasa de acuerdo a lo entendido por las mujeres que la sumisión femenina se aprende de las propias madres como una forma válida de relación con el mundo masculino, la subordinación se acata pero no se comparte, se acepta por miedo o por evitar conflictos que terminen en violencia, especialmente si están los hijos e hijas como testigos, además se agrega la creencia de que siendo sumisa se evita el abandono.

Los aprendizajes en el estilo de relacionarse se adquieren en la infancia, las abuelas entrevistadas reconocen haber crecido y formado a sus hijas en un entorno hostil y violento, siendo estas pautas traspasadas a sus hijas como una forma de enseñanza; se reconoce la brutalidad en el aprendizaje, connotándolo como negativo, lo que significa que no es un acto naturalizado, este reconocimiento provoca en las mujeres adultas sufrimiento, culpa, contradicciones y desasosiego que se trasmite transgeneracionalmente, sin embargo, es legitimado frente a ciertas conductas de las mujeres.

Por otra parte, el abuso sexual intrafamiliar es una situación abusiva que se evidencia en los relatos de las tres generaciones 
de mujeres de este estudio, se minimiza no porque se encuentre naturalizado, sino porque avergüenza, es validado a partir de las creencias asociadas en las mujeres adultas de que hay un mandato que cumplir con respecto a los deberes con la pareja, esta sumisión no es observable pues queda en el espacio privado. Las abuelas y las madres manifiestan al respecto, un conflicto interno frente a las agresiones sexuales; el dolor se acompaña de rebeldía, impotencia, impunidad que también se transmite. La rabia es la emoción más potente expresada por las mujeres adultas, son experiencias que vivencian como traumáticas y se trasmiten a las demás generaciones como un acto que no debe ser contado, pasando a conformar parte de los secretos de las familias; esta opción podría dar cuenta de variados aspectos que las mujeres adultas evalúan como pertinentes para el silenciamiento.

Se trasmite en la primera generación de abuelas, la resignación ante acontecimientos tan traumáticos, percibiendo que esta conducta sí se encuentra naturalizada en esta generación, siendo traspasada a las hijas de la misma forma. No obstante, todas las madres transmiten a sus hijas (nietas de la investigación) que no hay que guardar silencio ante estos actos, siendo todas ellas capaces de denunciar los abusos a sus hijas, considerando necesaria la reparación psicológica, por tanto en esta generación no está naturalizada. Es paradójico evidenciar cómo en algunas situaciones expresadas por las mujeres, se transmite a las generaciones de las hijas-nietas la importancia de no aceptar abusos de tipo sexual, enseñando a sus hijas que no son actos sanos y por lo tanto hay que decirlo y pedir ayuda para reparar el trauma; este discurso de las madres hacia las hijas, no ha sido transmitido hacia ellas por sus madres (abuelas de esta investigación); contrariamente a lo señalado, algunas abuelas conviven aún con los agresores de sus hijas, minimizando y justificando los hechos o no dando credibilidad a sus hijas. Por su parte estas hijas, hoy madres, no responsabilizan a sus madres (abuelas) de sus vivencias, tienden a justificarlas a partir del sufrimiento que han vivido y que sus progenitoras les han transmitido, asociado al temor de que sus madres (abuelas del estudio) podrían tener que enfrentar a los hombres y sufrir un nuevo abandono. Las madres han aceptado con pasividad la opción de sus progenitoras de continuar con sus parejas, hecho que refieren con rencor hacia el agresor, no hacia la madre, más bien es señalado como una consecuencia de lo vivido y por tanto, la incapacidad de las abuelas de haber sido protectoras 
se justifica provocando en algunas de las madres la necesidad de asumir un rol protector incluso con su propia madre.

Por otra parte, la misma decisión provocó la rabia y desilusión hacia su progenitora, dañando el vínculo entre ambas hasta el día de hoy. Cabe mencionar que las madres, a pesar del discurso que profesan con respecto a los derechos, al daño, la necesidad de pedir ayuda para sus hijas, no pudieron impedir que el patrón se repitiera con la tercera generación (nietas del estudio), y se movilizaron una vez develados los abusos. Se transmite por tanto, la aceptación de la condición de mujer víctima y su vulnerabilidad (abuelas) por sobre la madre protectora, esta sensación de desamparo, produce sufrimiento en las mujeres ante las escasas alternativas de cambio en sus vidas, se transmite la imagen de mujeres desamparadas, frente a esta profecía autocumplida, reconociendo que los cambios pasan por no repetir los patrones aprendidos en la infancia. Saben que es prioritario dejar de sufrir, por lo tanto como ser mujer implica sufrir, se instalan estas pautas en las nuevas generaciones aunque a ellas no les hayan dado los resultados que esperaban, la desesperanza se sigue transmitiendo. Es concordante lo anterior, con las experiencias vividas en la infancia y adolescencia por la imagen que se transmitió desde la familia de origen.

Con respecto de las mujeres adultas, éstas transmiten a sus hijas la incondicionalidad que debe existir hacia la madre, lo que implica priorizar a la familia, esto a pesar de conocer los hechos abusivos que vivieron sus hijas con sus respectivas parejas; se transmite de igual modo que la condición de madre está por sobre las vivencias; lo que corresponde a una contradicción constatada en la generación de las abuelas que exigen incondicionalidad al vínculo. Son las mujeres de la segunda generación (madres) las que se rebelan ante esta creencia, pues le adjudican grados de responsabilidad a sus madres en sus propios fracasos, porque se espera que las madres sean protectoras, lo contrario es fuente de tristeza, sin embargo, a pesar de su resentimiento permanecen junto a sus madres, por que lo integraron como creencia también transmitida.

Por su parte, las abuelas registran la ingratitud como sentimiento con respecto a sus hijas, se apropian del resentimiento como un sentimiento común y como una forma de relación entre madres e hijas, aceptando que frente a la imposibilidad de transformación de su situación, deben vivir con culpa y tristeza, expresada como se ha dicho, a través de la rabia, como emoción valorada. 
Existe otra creencia que ha sido transmitida, relativa a que verse expuesta a recibir violencia en espacios públicos, es una situación que no debe ser aceptada, experiencia vivenciada por las abuelas, madres y nietas adolescentes, todas de la misma línea generacional, lo que motivó el rechazo de parte de ellas, sin embargo, su reacción es distinta ante agresiones recibidas en el ámbito privado, estas son referidas con vergüenza y rabia pero parte de una relación familiar. Es relevante este punto, tomando en cuenta que ninguna estrategia utilizada por las mujeres para detener la violencia había inducido al cambio, porque al interior del hogar, por grave que esta sea, se tiende a minimizar; contrariamente la exposición pública genera emociones distintas que las hace tomar decisiones en muchas ocasiones drásticas, como separarse del agresor, transmitiendo a las nuevas generaciones la no aceptación de estos hechos, y la necesidad de escapar como alternativa de solución.

Además las abuelas trasmitieron a sus hijas que para salir de situaciones de abandono y maltrato era necesario conocer a un hombre y casarse, la pareja supone una vía de escapeal sufrimiento; en este contexto el hombre se transforma en el salvador, quien rescata del dolor. Si bien en la práctica las abuelas constataron que esta estrategia no fue una alternativa que modificó sus vidas y lo reconocen como un fracaso, no deja de ser un mecanismo de escape que las mujeres siguen validando y es transmitido del mismo modo a sus hijas y por éstas a las nietas.

En la generación de las abuelas se aprecia mayor aceptación a ciertas situaciones abusivas, no obstante, esa aceptación no necesariamente implica naturalización, pues lo viven con altos montos de desasosiego y rabia debido a la escasa posibilidad de modificar la situación. Las abuelas permanecen por más tiempo en situaciones dolorosas, su historia de vida, la desesperanza, el temor a los cambios les impide tomar decisiones. Son las abuelas las que trasmiten que la sumisión del género femenino favorece la unión familiar, no como algo que se valora sino como un ordenamiento dado, las madres lo aprendieron de las abuelas y ellas lo socializan con sus hijas. Sin embargo, al momento de buscar alternativas de escape, son las propias mujeres quienes se auto-generan las estrategias de salida de las situaciones abusivas, lo que se contradice con la transmisión de desesperanza aprendida.

Con respecto a las explicaciones que se dan las mujeres de las tres generaciones, recogido a través del análisis estructural semántico, 
se puede señalar que todas esperan alcanzar el objeto valor que es no sufrir, para esto cuentan con ciertos ayudantes; hombres, que les permitirán alcanzar el objetivo. Sin embargo, preexisten los oponentes, los adultos a cargo, la sensación de soledad y de culpa que intentará obstaculizar este proceso, los hombres serán en un primer momento la alternativa para el cambio a una mejor calidad de vida y la de sus hijos. El objeto valor que es dejar de sufrir, se alcanzará si se abandona la familia y se crea una nueva, el no logro del fin último se lo explican desde su propia identificación como mujeres sumisas, desesperanzadas y desprotegidas, desde su entorno más cercano son percibidas como mujeres cansadas, humilladas, enrabiadas y tristes, finalmente el objeto valor que es dejar de sufrir no se alcanza porque los hombres que las rescatarían pasan a conformar la categoría de oponentes. No alcanzan el objeto valor, sus hijos e hijas como remitentes no logran una mejor calidad de vida y tampoco lo logran las mujeres destinatarias, generando sentimientos de frustración.

En relación a la violencia y el maltrato, el objeto valor de todas las mujeres es dejar de vivir situaciones abusivas, desde sus explicaciones los ayudantes para lograr el fin son las propias mujeres, los hijos e hijas y mayor grado de autonomía e independencia. Los adversarios $u$ oponentes se conforman de los hombres, agresores, la cultura, el aprendizaje y las creencias, estos oponentes son potentes como la cultura que determina pautas y roles, además de un aprendizaje asociado a creencias y mitos que se transmite desde la infancia. Con respecto a los ayudantes con que cuentan las mujeres para alcanzar el objeto valor, estos se ven debilitados paulatinamente siendo interferidos por los adversarios, no permitiendo asegurar con esto el logro del objetivo de las mujeres que es dejar de vivir violencia, son las propias dinámicas violentas al interior del núcleo las que boicotean las estrategias, involucrando a los ayudantes y finalmente emplazándolos como adversarios.

Las percepciones de sí mismas de las mujeres como personas horrorizadas, humilladas, violentadas, no amadas e impotentes, se contrasta con la percepción de los otros que las ven como vulnerables, desprotegidas, desesperanzadas y violentas, desde estas explicaciones las mujeres reconocen la imposibilidad de alcanzar el objeto valor y por lo tanto, no mejoran la calidad de vida de sus hijos que son los remitentes de su acción, ni de ellas mismas como destinatarias, quedando en la misma condición 
que es percibida como parte de su esencia y se transmite del mismo modo.

Las mujeres se explican el no logro del objeto valor que es ser protectoras, por la presencia de oponentes como; violencia, situación de pobreza, creencias y pasividad. Los ayudantes con los que cuentan son pedir ayuda externa y reconocer la negligencia como un acto abusivo. Las explicaciones que se dan las mujeres, tienen relación con su pasividad, que les impide movilizarse hacia el objeto valor que es ser protectoras, porque están centradas en sentirse víctimas, sacrificadas y preocupadas por mantener unida la familia. Sin embargo, su entorno las cataloga como malas madres, castigadoras, irresponsables, abandonadoras, indiferentes y poco afectivas. La estrategia de acción para alcanzar el objeto valor son las abuelas que acompañan y ellas como madres que reparan. Finalmente la presencia de los adversarios, impide a las mujeres alcanzar el objeto valor de ser protectoras y permitir mejorar la calidad de vida de sus hijos e hijas remitentes y de ellas como destinatarias.

\section{Conclusiones}

Los mitos subyacentes en los relatos de las mujeres, dicen relación con las creencias y con el discurso social con respecto a lo masculino y femenino, que establece ciertas normas y pautas de relacionarse a partir del género: la desigualdad y asimetría en las relaciones, lo que se ve reflejado en todos los relatos de las mujeres entrevistadas, como aprendizaje desde sus familias de origen. Este sistema de creencias está dado por un ordenamiento social, desde un macro sistema que legitima la desigualdad y la subordinación, avalando la violencia como forma de enseñanza, validando las creencias como las expresadas en las entrevistas de las mujeres, siendo la más arraigada la referida a que la violencia sería una forma de enseñar perteneciente al ámbito de lo privado.

De este modo, este estilo de relación se perpetúa a través de las generaciones, se evidencia claramente en las experiencias descritas por las mujeres entrevistadas, que estos estilos de relacionarse transmitidos transgeneracionalmente, se instalan como mandatos en las familias. Estos aprendizajes y creencias se traspasan de generación en generación, siendo fiel reflejo de esta realidad, las vidas narradas por las mujeres entrevistadas. 
Se confirman los supuestos desde los que partió este estudio, al considerar que efectivamente se pueden alterar las maneras de establecer los vínculos al interior de una familia a través de las generaciones, si existen situaciones abusivas al interior de una familia que están sustentadas firmemente por creencias y mitos, los cuales se van traspasando de generación en generación. Es esta dimensión transgeneracional la fuerza que naturaliza y reproduce la violencia de género.

Considerando lo anterior se sugiere realizar una intervención integral con las familias a través de equipos profesionales interdisciplinarios, dado que la reparación y la re-significación a nivel individual no asegura la no repitencia de situaciones abusivas, como así también, es necesario ir desconstruyendo los mitos que han servido como brújulas familiares, que han dirigido generacionalmente las conductas de los integrantes de un sistema familiar.

\section{Referencias Bibliográficas}

BARUDY, J. (2001). Maltrato Infantil: Ecología Social; Prevención y Reparación. Galdoc. Santiago de Chile.

BOSZORMENKYI-NAGY, I. (1982). Lealtades Invisibles. Amorrourtu. Buenos Aires.

BOWEN, M. (1981). De la familia al individuo. Paidós. Barcelona.

ITURRIETA, S. (2001). Conflictos familiares ¿cómo resolverlos? Centro de educación a Distancia. Universidad Católica del Norte. Chile.

LARRAIN, S. (2002). La violencia en la familia y transmisión de pautas de comportamiento. Impreso en Naciones Unidas. Santiago de Chile.

PALMA, I. (2007). Caracterización Teórico Metodológica de las Intervenciones Grupales con Mujeres Víctimas de Violencia en la Pareja. Universidad de Chile. Santiago de Chile.

RAVAZZOLA, C. (1997). Historias Infames: el maltrato en las relaciones. Paidós. Buenos Aires.

GRACIA Y MUSITU (2000). En: Iturrieta, S. (2001). Conflictos familiares ¿cómo resolverlos? Centro de educación a Distancia. Universidad Católica del Norte. Chile.

KLEIN Y WHILE (1996). En: Iturrieta, S. (2001). Conflictos familiares ¿cómo resolverlos? Centro de educación a Distancia. Universidad Católica del Norte. Chile. 